\title{
Effect of lateral connections on the accuracy of the population code for a network of spiking neurons
}

\author{
Mona Spiridon and Wulfram Gerstner \\ Center for Neuromimetic Systems, Swiss Federal Institute of Technology, Lausanne, EPFL-DI \\ CH-1015 Lausanne EPFL, Switzerland
}

Received 15 August 2000, in final form 23 March 2001

Published 15 August 2001

Online at stacks.iop.org/Network/12/409

\begin{abstract}
We study how neuronal connections in a population of spiking neurons affect the accuracy of stimulus estimation. Neurons in our model code for a onedimensional orientation variable $\phi$. Connectivity between two neurons depends on the absolute difference $\left|\phi-\phi^{\prime}\right|$ between the preferred orientation of the two neurons. We derive an analytical expression of the activity profile for a population of neurons described by the spike response model with noisy threshold. We estimate the stimulus orientation and the trial-to-trial fluctuations using the population vector method. For stationary stimuli, uniform inhibitory connections produce a more reliable estimation of the stimulus than shortrange excitatory connections with long-range inhibitions, although the latter interaction type produces a sharper tuning curve. These results are consistent with previous analytical studies of the Fisher information.
\end{abstract}

\section{Introduction}

The response of most neurons in the central nervous system shows a high degree of variability (Werner and Mountcastle 1965, Shiller et al 1976, Vogels et al 1989, O'Keefe et al 1997). Since neuronal noise has a detrimental impact on information processing, redundancy is needed to average out the noise. The primary visual cortex, for example, is organized in columns composed of neurons that respond preferentially to the same stimulus orientation (Hubel and Wiesel 1968, 1974). Using a population of neurons with similar properties to encode information has the advantage of reducing the noise. On the other hand, if neurons in the population are connected to each other or share input, they cannot be treated as independent. Because it is likely that nearby neurons share more common inputs than distant neurons, correlated variability is expected to decrease with interneuronal distance (Braitenberg and Schuz 1991, Zohary et al 1994, Douglas et al 1995). Although the experimentally measured correlation coefficients are quite small, correlated variability is thought to limit the information processing capacity of neuronal ensembles (Zohary et al 1994). However, several researchers have shown that the effect of correlated noise is not always harmful to information processing 
(Snippe and Koenderink 1992, Abbott and Dayan 1999, Yoon and Sompolinsky 1998). Snippe and Koenderink (1992) have indicated that, if the spatial range of correlated noise is larger than the receptive field width of neurons, it can lead to improvement of the discrimination thresholds in psychophysical tasks. Recently, Abbott and Dayan (1999) and Yoon and Sompolinsky (1998) have used Fisher information to provide an estimation of the accuracy of the population code using neurons described by a rate model. Under the hypothesis that the neuronal activity follows a multivariate Gaussian distribution, they have shown some effects of the shape of the correlation matrix on the population code accuracy. For instance, Yoon and Sompolinsky (1998) have found that negative correlations enhance the Fisher information relative to the uncorrelated case, whereas positive correlations almost always decrease it. Only in the extreme case of very long correlations can positive correlations produce a higher information value than in the case of an uncorrelated population. Thus, depending on the form of the correlation matrix, correlated variability can either increase or decrease the information capacity in comparison to the uncorrelated case.

In these studies correlated noise was introduced explicitly into the model by the definition of a correlation matrix (Snippe and Koenderink 1992, Abbott and Dayan 1999, Yoon and Sompolinsky 1998). Nevertheless, these correlations must somehow be generated inside the neural network. Correlations induced by common input have been considered in other studies (Shadlen and Newsome 1998). In this paper, we explore the possibility that correlated noise originates from the lateral connections inside the neural network. We study how correlations induced by lateral connections affect the accuracy of the population code. We compare two types of interactions: uniform inhibition (homogeneous interactions) and short-range excitation with long-range inhibition (modulated interactions). It has been suggested that these interaction types play an important role in the mechanisms for orientation selectivity in the primary visual cortex. Indeed, V1 neurons receive a broad input from the LGN but their tuning curve is much sharper. Several models have been proposed to explain the sharpening of the tuning curve in V1. Some models suggest that orientation selectivity is mainly caused by lateral inhibition (Ferster and Koch 1987, Wörgötter and Koch 1991, Wehmeier et al 1998) whereas other authors have proposed that orientation tuning is the result of short-range excitations together with longer range inhibitions (Ben-Yishai et al 1995, Somers et al 1995), producing a Mexican-hat-shaped interaction between orientation columns.

As in previous studies of Ben-Yishai et al (1995) and Hansel and Sompolinsky (1998), we use a one-dimensional population of spiking neurons, where each neuron codes for a particular orientation of the stimulus. In section 2 , the model is defined. In section 3 , an analytical expression of the activity profile is derived. In section 4 , we define how we assess the accuracy of the estimation and discuss how lateral interactions induce correlation.

\section{The model}

\subsection{The network model}

We consider a population of $N$ interconnected neurons evenly distributed on a one-dimensional line. Each neuron $i$ codes for a particular scalar feature $\phi_{i}$. The feature $\phi$ can represent any external physical variable with which neural activity is correlated. For simplicity we will assume periodic boundary conditions, and interpret $\phi$ as the orientation of a stimulating light bar. All functions of $\phi$ are periodic functions with period $\pi$.

Each neuron $i$ is selective for a range of orientations and responds maximally when a particular value $\phi_{i}$ of the stimulus orientation, called the preferred orientation, is present. The values of preferred orientations $\phi_{i}$ are uniformly distributed among the neurons in the interval 


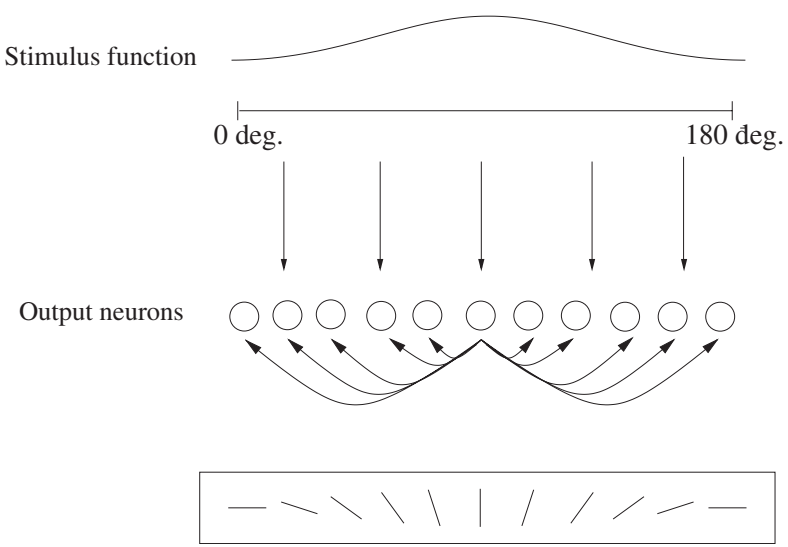

Figure 1. Sketch of the network architecture. The input stimulus potential (equation (2)) is broad and periodic (top). The neuronal network is fully connected. Here, we have represented only the lateral connections from one neuron (middle). The connection strengths are described by equation (6). Neurons are characterized by their preferred orientation (bottom).

$0<\phi_{i} \leqslant \pi$. The total input $h_{i}$ to each neuron consists of two parts, i.e. an external input $h_{i}^{\text {ext }}$ (stimulus) and the input $h_{i}^{\text {int }}$ from the other neurons of the network (figure 1):

$$
h_{i}(t)=h_{i}^{\text {int }}(t)+h_{i}^{\text {ext }}(t) .
$$

If the stimulus has orientation $\phi_{0}$, the external input to neuron $i$ is represented by a Von Mises function (Fisher 1993):

$$
h_{i}^{\text {ext }}\left(\phi_{0}\right)=\frac{h^{\max }}{\exp \left(\alpha^{-1}\right)} \exp \left\{\alpha^{-1} \cos \left[2\left(\phi_{0}-\phi_{i}\right)\right]\right\} .
$$

This function is similar to a Gaussian but is $\pi$-periodic. The parameter $\alpha$ corresponds to the width of the function. We choose a large value of $\alpha$ so that the stimulus is broadly tuned. The first term of equation (2) normalizes the function, so that the maximum amplitude is equal to $h^{\max }$. A neuron $i$ responds maximally when the orientation of the input stimulus $\phi_{0}$ is equal to its preferred orientation $\phi_{i}$.

The lateral input $h_{i}^{\text {int }}$ is characterized by the coupling strength $J_{i j}$ and the time course $\varepsilon_{i j}(t)$ of the postsynaptic potential

$$
h_{i}^{\mathrm{int}}(t)=\frac{1}{N} \sum_{j=1}^{N} \sum_{t_{j}^{(f)}} J_{i j} \varepsilon\left(t-t_{j}^{(f)}\right)
$$

where the sum runs over all firing times $t_{j}^{(f)}$ and over all neurons in the network. Note that we have taken the interaction strength to scale inversely with the total number of neurons $N$. The kernel $\varepsilon$ can be chosen arbitrarily. Here, we take

$$
\varepsilon(s)=\frac{1}{\tau_{\mathrm{s}}-\tau_{\mathrm{m}}}\left[\exp \left(-\frac{s}{\tau_{\mathrm{s}}}\right)-\exp \left(-\frac{s}{\tau_{\mathrm{m}}}\right)\right] \mathcal{H}(s)
$$

where $\tau_{\mathrm{s}}$ and $\tau_{\mathrm{m}}$ are respectively the synaptic and membrane time constants. To ensure causality we have added the Heaviside function $\mathcal{H}(\cdot)$ with $\mathcal{H}(s)=1$ for $s>0$ and 0 otherwise. $\varepsilon$ is normalized so that $\int_{0}^{\infty} \varepsilon(s) \mathrm{d} s=1$. For $\tau_{\mathrm{m}}=\tau_{\mathrm{s}}=\tau$, equation (4) reduces to

$$
\varepsilon(s)=\frac{s}{\tau^{2}} \exp \left(-\frac{s}{\tau}\right) \mathcal{H}(s)
$$




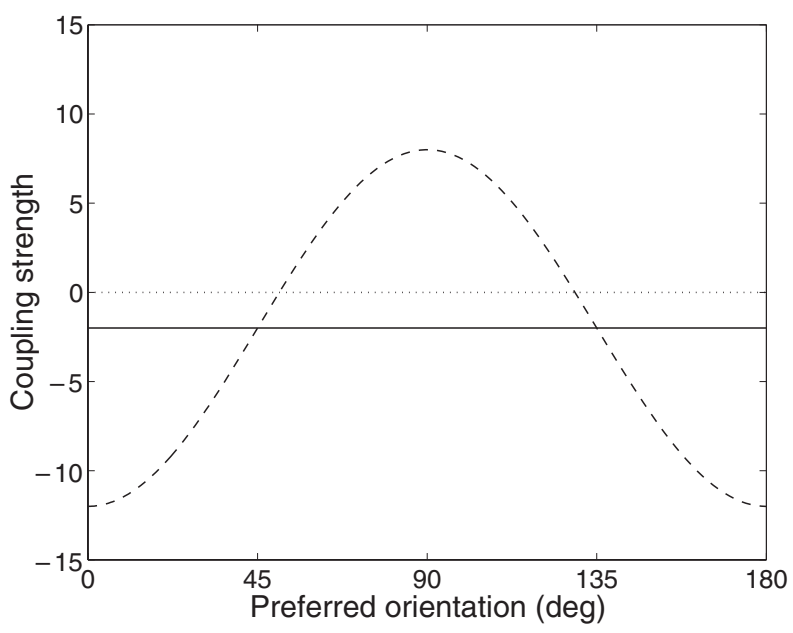

Figure 2. Coupling strength function for a neuron at $90^{\circ}$ preferred orientation as a function of the preferred orientation. Two types of interaction described by equation (6) are plotted here. The solid line represents uniform inhibition $\left(J_{0}=-2, J_{2}=0\right)$ and the dashed curve shows modulated interaction $\left(J_{0}=-2, J_{2}=10\right)$.

For the sake of simplicity, we assume that neurons can have both excitatory and inhibitory connections with each other. This coupling model is of course not biologically plausible. However, Ben-Yishai et al (1995) and Hansel and Sompolinsky (1998) have shown that many qualitative properties of the stationary states remain when the onetype population is replaced by a population with excitatory and inhibitory neurons. The interaction strength $J_{i j}$ is assumed to be strongest in magnitude for neurons with similar preferred orientations. This hypothesis is consistent with the anatomical and physiological evidence available in the primary visual cortex (Ferster 1986, Martin 1988). In order to describe the orientation dependence of the coupling strength, we choose the type of interaction used by Ben-Yishai et al (1995) to study the properties of the orientation tuning curves in the visual cortex. It is expressed using the first two terms of the Fourier series

$$
J_{i j}=J\left(\phi_{i}-\phi_{j}\right)=J_{0}+J_{2} \cos \left(2\left(\phi_{i}-\phi_{j}\right)\right) .
$$

Only even terms are non-null, as the period of $J$ is $\pi$. The coupling equation is represented in figure 2 for two different values of $J_{2}$.

\subsection{The neuronal model}

The membrane potential $u_{i}$ of neuron $i$ describes the state of the neuron. In the spike response model, the membrane potential is given by (Gerstner 1995):

$$
u_{i}(t)=\eta\left(t-\hat{t}_{i}\right)+h_{i}(t)
$$

where $h_{i}(t)$ is the total input (equation (1)) described in section 2.1 and $\hat{t}_{i}$ is the firing time of the last spike of neuron $i$. The function $\eta$ describes the refractory period following a spike. We use

$$
\eta(s)=-\eta_{0} \exp \left(-\frac{s-\delta_{\mathrm{abs}}}{\tau_{\mathrm{m}}}\right) \mathcal{H}\left(s-\delta_{\mathrm{abs}}\right)+K \mathcal{H}\left(\delta_{\mathrm{abs}}-s\right) \mathcal{H}(s)
$$


where $\delta_{\mathrm{abs}}$ is the absolute refractory period, $K$ is a large negative constant and $\tau_{\mathrm{m}}$ is the membrane time constant. We set $\eta_{0}=1$.

In order to reproduce the variability in the firings of cortical neurons, we add noise to the neuronal model. To do so, we introduce an escape rate $\rho$ that depends on the difference between the present value of the membrane potential $u(t)$ and the threshold $\vartheta$ :

$$
\rho_{h_{i}}\left(t \mid \hat{t}_{i}\right)=f[u(t)-\vartheta]=f\left[\eta\left(t-\hat{t}_{i}\right)+h_{i}(t)-\vartheta\right] .
$$

The second equality follows from (7). The escape rate $\rho$ is implicitly time dependent, since the membrane potential $u(t)$ varies over time. The choice of the function $f$ is arbitrary; since the results that we have obtained are qualitatively independent of the form of the escape rate, we use for simplicity a piecewise linear function

$$
\rho=\gamma_{0} \cdot(u-\vartheta) \mathcal{H}(u-\vartheta)
$$

where $\gamma_{0}$ is a parameter that determines the noise level. The higher the value of $\gamma_{0}$ the lower the noise. It should be noted that with an appropriate choice of $f$, the escape rate $\rho$ can reproduce, to a high degree of accuracy, noise due to stochastic spike arrival (Plesser and Gerstner 2000).

Given the escape rate function, we can calculate the survivor function, that is the probability that the neuron, after having emitted a spike at $\hat{t}_{i}$, will not emit a spike up to time $t$ (Cox 1962, Gerstner and Van Hemmen 1992, Gerstner 2000):

$$
S_{h_{i}}\left(t \mid \hat{t}_{i}\right)=\exp \left[-\int_{\hat{t}_{i}}^{t} \rho_{h_{i}}\left(s \mid \hat{t}_{i}\right) \mathrm{d} s\right] .
$$

The survivor function obviously depends on $\hat{t}_{i}$, the last firing time of neuron $i$. The lower index $h_{i}$ in the survivor function is intended to remind the reader that $S_{h_{i}}\left(t \mid \hat{t}_{i}\right)$ also depends on the input potential $h_{i}(t)$.

\section{Activity profile}

The activity profile describes the firing rate of the neurons as a function of their preferred orientations. Since the network is completely axisymmetric, the coupling between two neurons only depends on the absolute difference $\left|\phi-\phi^{\prime}\right|$ between their preferred orientation. Therefore, the form of the activity profile is identical to the form of the tuning curve of a single neuron. For a time-stationary state the activity profile of a large population of neurons $(N \rightarrow \infty)$ can be obtained from a set of self-consistent equations. Indeed, in a stationary state,

$$
h_{i}^{c}=\frac{1}{N} \sum_{j=1}^{N} J_{i j} r_{j}+h_{i}^{\mathrm{ext}}
$$

where we have used the fact that $\int_{0}^{\infty} \varepsilon(s) \mathrm{d} s=1$. Equation (12) becomes exact in the limit of $N \rightarrow \infty$; see appendix $\mathrm{B}$. The mean firing rate $r_{i}$ for neuron $i$ can be calculated from the survivor function (Gerstner and Van Hemmen 1992)

$$
r_{i}=\left[\int_{0}^{\infty} S_{h_{i}^{c}}(s) \mathrm{d} s\right]^{-1},
$$

where for a constant potential $h_{i}(t)=h_{i}^{c}$, the survivor function $S_{h_{i}^{c}}\left(t \mid \hat{t}_{i}\right)$ depends only on the time difference $t-\hat{t}_{i}$. So far, analytical expressions for the activity profile have been obtained with rate models (Ben-Yishai et al 1995, Hansel and Sompolinsky 1998) and integrate-and-fire models in the absence of internal noise (Laing and Chow at press). Equations (12) and (13) give a theoretical solution for the activity profile in a network of spiking neurons with internal noise. Figure 3 shows that the activity profile resulting 

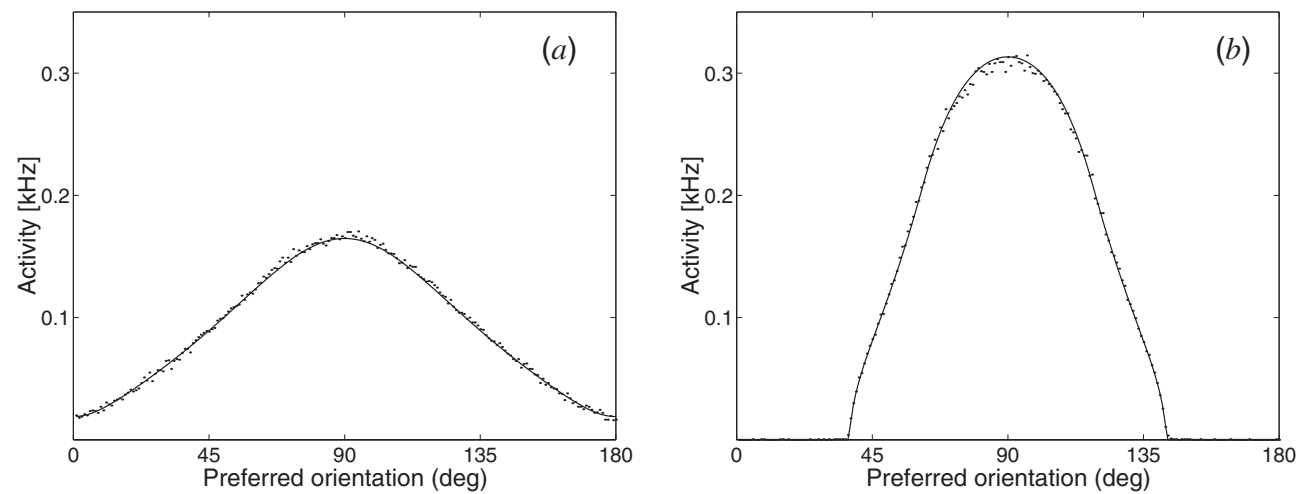

Figure 3. Activity profile obtained from simulations (dots) compared with theory (solid curve). The rate in simulations is estimated from spike counts over $2 \mathrm{~s}$ following the onset of the stimulus with a population of 200 neurons. The amplitude of the external stimulus $h^{\max }=2$, the width $\alpha=4$ and the orientation is $\phi_{0}=90^{\circ}$, cf equation (2). The values for the interaction strength are (a) $J_{0}=-2$ and $J_{2}=0$ and $(b) J_{0}=-2$ and $J_{2}=10$. Neurons have an absolute refractory period of $2 \mathrm{~ms}$ and the synaptic and membrane time constants are both equal to $4 \mathrm{~ms}$. The neuronal noise is described by a linear escape rate model with $\gamma_{0}=1$.

from these equations matches to a high degree of accuracy the one obtained by simulating a population of spiking neurons described by the model in section 2 . The sharper tuning curve obtained with the local excitation with long-range inhibition can be understood as the result of the similarity between the shape of the tuning curve and that of the connection pattern: a neuron in the centre of the stimulus $\left(\phi=90^{\circ}\right)$ strongly stimulates its neighbours (and therefore reinforces the peak) while it inhibits neurons with orthogonal preferred orientations.

\section{Accuracy of the stimulus estimator}

An estimation of the stimulus orientation $\phi^{\text {est }}$ is obtained using the population vector method (Georgopoulos et al 1983, Salinas and Abbott 1994). The accuracy of the estimator is measured by the variance of the trial-to-trial fluctuations. Because the variable $\phi$ is distributed on a circle, we use the circular standard deviation (CSD) to measure the fluctuations in the orientation (Mardia 1972) (see appendix A). The CSD of the orientation estimator is computed for different time window durations $T$, starting from the initiation of the stimulus. For stationary stimuli, the longer the duration of the time window, the better the estimation. For long time window duration $(>100 \mathrm{~ms}$ ) the CSD decreases approximatively with $1 / \sqrt{T}$. The stimulus is initiated at $t=0 \mathrm{~ms}$. Before the start of the stimulus all neurons are silent ${ }^{1}$.

\subsection{Effect of lateral connections}

We would like to understand how the lateral connections affect the accuracy of the population code. To do so we compare the CSD obtained using uniform inhibition (equation (6) with $J_{2}=0$ ) and the one obtained using modulated interactions (equation (6) with $J_{2}>0$ ). Figures 4(a) and $(b)$ represent the theoretical activity profiles and the CSD obtained by these two interaction types. We find that although short-range excitation sharpens the tuning curve

1 We do not take into account any spontaneous activity for $t<0$, in order to simplify the comparison of the CSD for different parameters. Indeed, the spontaneous activity would change each time we change the value of a parameter. 

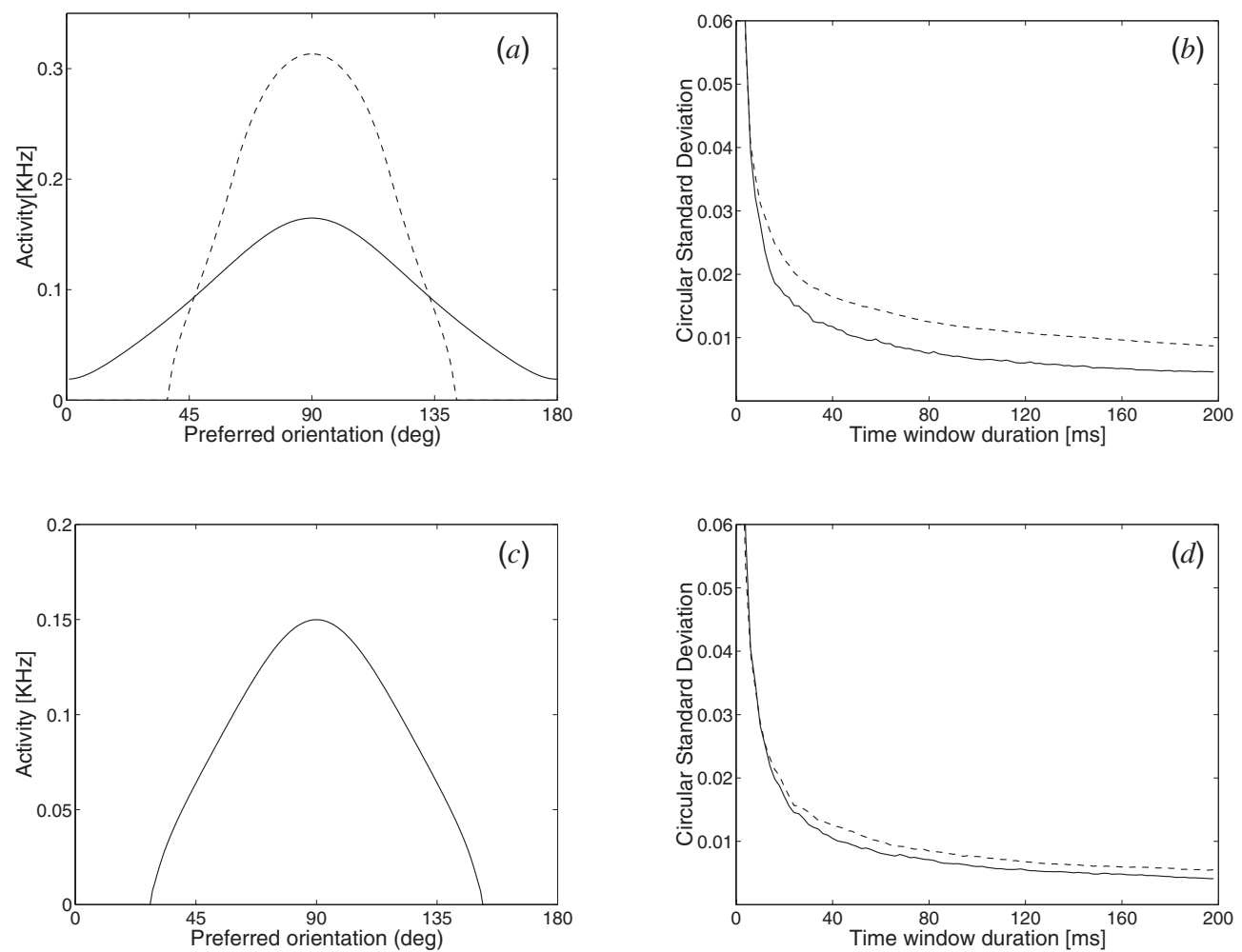

Figure 4. (a) Activity profile and (b) CSD for a network with uniform inhibition $\left(J_{0}=-2\right.$, $J_{2}=0$ ) (solid curve) and for a network with short-range excitation and long-range inhibition $\left(J_{0}=-2, J_{2}=10\right)$ (dashed curve). In the two cases, the stimulus amplitude is equal to $h^{\max }=2$. We see that even though the tuning curve is shaper for $J_{2}=10$, the estimation is worse. (c) Activity profile. The stimulus amplitude $h^{\max }$ has been adjusted in order to obtain the same activity profile for the two types of interaction. The activity profiles for uniform inhibition $\left(J_{0}=-5, J_{2}=0, h^{\max }=1.96\right)$ and modulated interactions $\left(J_{0}=0, J_{2}=2, h^{\max }=1.55\right)$ lie on top of each other and are indistinguishable. (d) CSD for uniform interactions (solid curve) and modulated interactions (dashed curve). In all cases the orientation of the stimulus is $90^{\circ}$ and the CSD is calculated from 500 trials with a population of 200 neurons. The network and neuronal parameters are the same as in figure 3 .

(figure 4(a)), the reliability of the estimation decreases (figure 4(b)). Modulated interactions produce a less reliable estimation even when the activity profile generated by modulated interactions is exactly identical to the one obtained by uniform inhibition (figures $4(c)$ and $(d)$ ). The spatial dependence of the interactions therefore appears to be important in the determination of the accuracy of the population code.

In order to better understand what determines the accuracy of the estimator, we compare the standard deviation obtained by the population vector method with the one obtained using Fisher information. The Fisher information, through the Cramer-Rao bound, gives a measure of the lowest variance of an unbiased estimator (Blahut 1988).

$$
\sigma_{\phi}^{2} \geqslant \frac{1}{I(\phi)}
$$

where $I(\phi)$ is the Fisher information for orientation $\phi$. If the neuronal activities follow a Gaussian distribution, the Fisher information can be expressed as a function of the covariance 

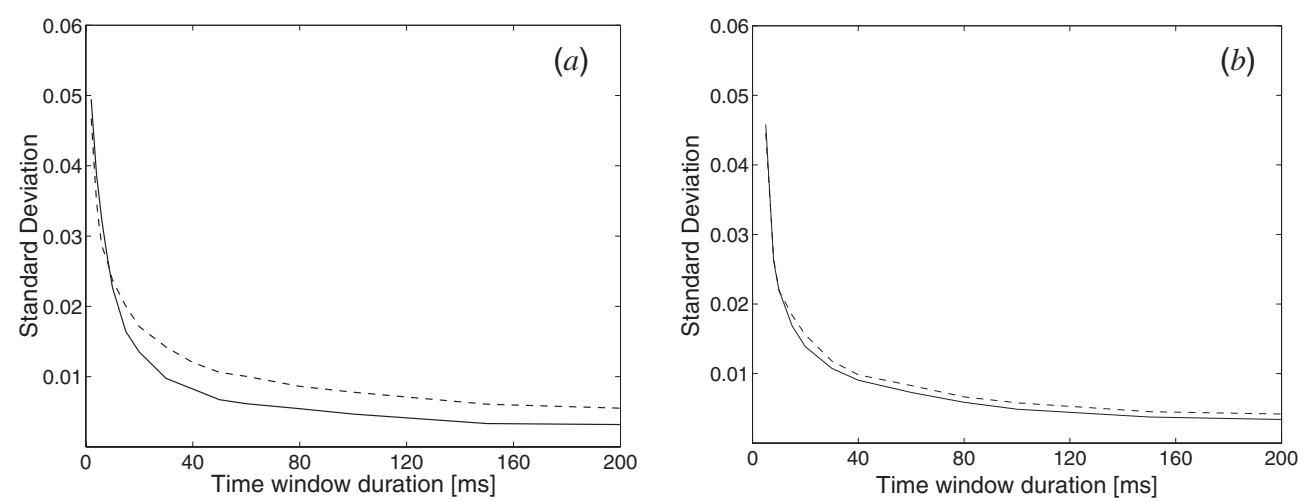

Figure 5. Lower bound of the standard deviation using Fisher information for a network with uniform inhibition (solid curve) and for a network with short-range excitation and long-range inhibition (dashed curve). The coupling parameters are the same as in figure $4(b)$ for $(a)$ and in figure $4(d)$ for $(b)$. The covariance matrix has been estimated from a simulation over 500 trials.

matrix and of the tuning curve derivative (Abbott and Dayan 1999, Yoon and Sompolinsky 1998):

$$
I(\phi)=\sum_{i, j} f_{i}^{\prime}(\phi) C_{i j}^{-1} f_{j}^{\prime}(\phi)
$$

where $f_{i}(\phi)$ represents the tuning function of neuron $i$ and $f_{i}^{\prime}(\phi) \equiv \frac{\partial f_{i}(\phi)}{\partial \phi}$. The covariance matrix $C_{i j}(\phi)$ of the firing rates $\left(r_{i}, r_{j}\right)$ is defined as follows:

$$
C_{i j}(\phi)=\frac{1}{K} \sum_{k=1}^{K}\left(r_{i}^{k}-f_{i}(\phi)\right)\left(r_{j}^{k}-f_{j}(\phi)\right)
$$

where $r_{i}^{k}$ is the firing rate of neuron $i$ on trial $k$ and $K$ is the total number of trials. We measure the covariance matrix (equation (16)) from simulations over $100 \mathrm{~ms}$ (figure 6) and calculate the Fisher information via equation (15). This gives a lower bound on the variance (cf equation (14)). Due to the refractory period, the distribution in spike counts of our model is well approximated by a Gaussian distribution even for short time window durations. We found that the standard deviation calculated using Fisher information gives slightly lower values than the population vector method as it should be, since Fisher information gives the optimal estimator. However, we find that the effect of a network or neuronal parameter on the accuracy of the stimulus estimation is qualitatively similar for both methods (figure 5). This means that the measure of the standard deviation is mostly independent of the decoding method.

The Fisher information allows an interpretation of our results. Equation (15) shows that the accuracy of the population code is determined by (i) the derivative of the tuning curve and (ii) the correlation matrix. In the case that the two tuning curves are the same as in figure 4(c), differences in Fisher information can only be generated by the second term, i.e. negative correlations induced by inhibitory interactions are advantageous to the accuracy of the population code. Figure 5(b) confirms that correlations induced by uniform inhibition lead to a lower value of the Fisher information than those induced by modulated interactions.

Figure 6 represents the covariance matrix for the two type of interactions (uniform inhibition and by modulated interactions). The diagonal terms have been removed for the purpose of visualisation. For uniform inhibitory interactions, the non-diagonal terms of the 

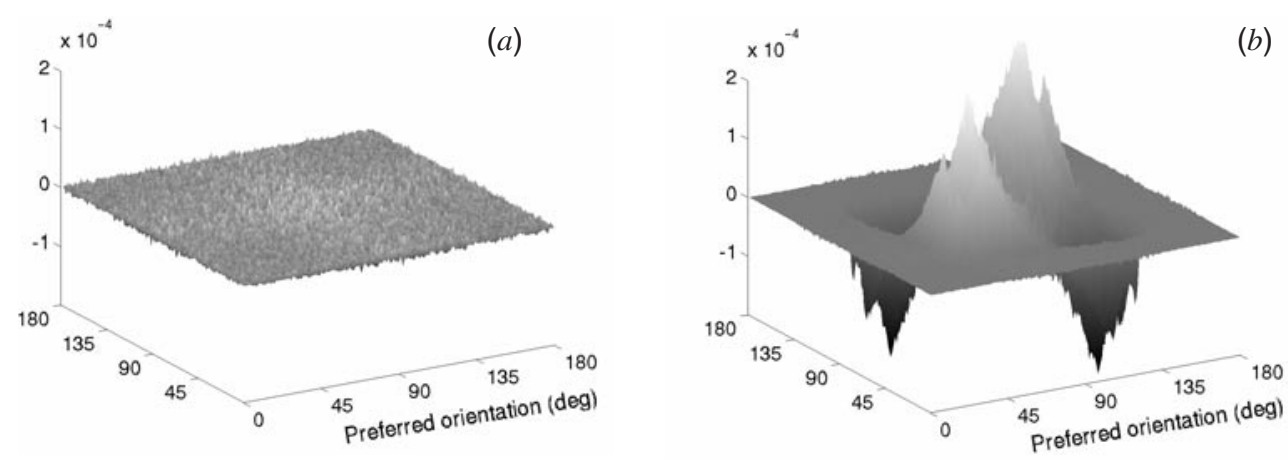

Figure 6. Covariance matrix of the firing rates for network of 200 neurons with uniform inhibition $\left(J_{0}=-2, J_{2}=0\right)(a)$ and modulated interactions $\left(J_{0}=-2, J_{2}=10\right)(b)$. A stimulus is applied with $\alpha=4, h^{\max }=2$ and $\phi_{0}=90^{\circ}$. The values on the diagonal have been removed for the purpose of visualization. The covariance matrix is estimated from a simulation over $100 \mathrm{~ms}$.

covariance matrix are uniformly negative but of very low amplitude ${ }^{2}$ (figure $6(a)$ ); this remains true even for very strong inhibition (Spiridon 2000). On the other hand, correlated noise produced by modulated interactions is positive for nearby neurons and negative between two distant neurons. Moreover, the same pair of neurons can have different correlations, depending on the type of stimulus. This can be seen in figure $6(b)$. Due to the complete symmetry of the system, measuring correlation between a given pair of neurons while changing the stimulus orientation would correspond to measuring correlations for different pairs of neurons (separated by the same preferred orientation difference) for a given stimulus orientation. Compare for example in figure $6(b)$ the pair of neurons at $45^{\circ}$ and $50^{\circ}$ and the pair at $90^{\circ}$ and $95^{\circ}$. Even though lateral interactions are the same, correlations are quite different. The effect of a correlation matrix as generated by this type of interaction on the Fisher information has not been studied yet analytically. Yoon and Sompolinsky (1998) have shown that local positively correlated noise decreases the estimation reliability. In our case, the estimation reliability is not only decreased by locally positively correlated noise but also by the negatively correlated noise between neurons with dissimilar preferred orientation. Indeed, we have estimated that the accuracy is improved by about $20 \%$ when the negatively correlated noise between distant neurons is removed and only the local positive correlations are retained.

\section{Discussion}

In contrast to previous work (Ferster and Koch 1987, Ben-Yishai et al 1995, Somers et al 1995), we were not interested in the shape of the tuning curve, but rather in correlations. In particular, we did not optimize parameters so as to obtain realistic profiles and we did not discuss how the form of the tuning curve changes with stimulation. Instead, we have studied how the accuracy of a population code depends on the lateral connection of a one-dimensional population of spiking neurons with periodic coupling. We have derived an analytical expression for the activity profile for stationary stimuli in a network of spiking neurons with internal noise. We have also studied the CSD of the stimulus orientation, estimated from simulations using the population vector method. We have shown that sharp activity profiles do not necessarily yield

2 Mathematically, a covariance matrix cannot have only large negative values, because it must be positive-definite. This explains why, even with strong uniform negative couplings, the amplitude of the negative correlations remains quite small. 
a better estimation accuracy (Brunel and Nadal 1998, Pouget et al 1999). By comparing the CSD between a network with uniform inhibition and a network with short-range excitation and long-range inhibition we have found that the latter interaction produces a less reliable stimulus estimation. Modulated interactions generate correlated noise that is positive for nearby neurons and negative for distant ones and this type of correlated noise reduces the accuracy of the orientation estimate. We have also simulated networks without any lateral connections (Spiridon 2000). The results are qualitatively similar to those obtained with uniform inhibition with a slightly lower value of the accuracy.

The effect of lateral connections on the stimulus estimation reliability can also be understood with intuitive arguments: a neuron $k$ with a preferred orientation different from the stimulus orientation may increase its activity as a result of its intrinsic noise. For modulated interaction, this will also increase the activity of its neighbouring neurons because of the local excitatory inputs. On the other hand, the activity of distant neurons will decrease because of the long range inhibition. Thus, the total effect is that the instantaneous activity profile will slightly shift towards the preferred orientation of neuron $k$. In contrast, with uniform inhibition, the spontaneous increase in the activity of a given neuron will not develop further, as neurons close to the orientation of the stimulus will send it a strong inhibitory signal. This simple reasoning shows that statistical fluctuations are amplified by a network with short-range excitatory connections, and suppressed by a network with uniform inhibitory connections.

Our studies show that uniform inhibition is more advantageous than modulated interactions, if stimuli are to be represented as accurately as possible. Stimulus representation is, of course, only one among many possible tasks of the nervous system. For a working memory task, for example, modulated interactions with local excitation are useful to stabilize activity patterns that have been triggered by an external stimulus (Redish et al 1996, Camperi and Wang 1998).

\section{Appendix A}

The performance of the population vector in estimating the orientation is measured by the variance of the trial-to-trial fluctuations. Special treatment is required to describe the variability of a directional measure such as the stimulus angle. Because the variable $\phi$ is distributed on a circle, we use the CSD to measure the fluctuations in the orientation.

Suppose we have a set of angles $\phi_{k}, k=1 \ldots K$ in a range of $[0 ; \pi]$. We calculate

$$
C=\sum_{k=1}^{K} \cos 2 \phi_{k}, \quad S=\sum_{k=1}^{K} \sin 2 \phi_{k}, \quad R^{2}=C^{2}+S^{2} .
$$

The measure of the circular variance is given by

$$
V=1-[1-(1-R / K)]^{1 / 4} .
$$

The CSD is defined as

$$
\mathrm{CSD}=[-2 \log (1-V)]^{1 / 2}
$$

where $\log$ is the natural logarithm. If the variance is small, equation (19) reduces to

$$
\mathrm{CSD}=(2 V)^{1 / 2} .
$$

A theoretical justification of these results is given in Mardia (1972). The CSD as defined in equation (19) is used to assess the reliability of the angle estimation. 


\section{Appendix B}

We start from equation (3) and write it in the form

$$
h_{i}^{\mathrm{int}}(t)=\frac{1}{N} \sum_{j=1}^{N} J\left(\phi_{i}-\phi_{j}\right) \sum_{t_{j}^{(f)}} \int_{0}^{\infty} \varepsilon(s) \delta\left(t-t_{j}^{(f)}-s\right) \mathrm{d} s .
$$

We will show that for a large number of neurons $N \gg 1$ equation (21) approaches in a stationary state a constant value $h_{i}^{\text {int }}=N^{-1} \sum_{j} J\left(\phi_{i}-\phi_{j}\right) r\left(\phi_{j}\right)$ where $r\left(\phi_{j}\right)$ is the mean firing rate of a neuron with preferred orientation $\phi_{j}$.

To do so, we will think of our $N$ neurons as being organized in a finite number of distinct populations. Within each population neurons are assumed to be homogeneous. Only at the end will we drop the assumption of separate populations; see e.g. Gerstner (1995). Since the preferred angles $\phi_{j}$ are evenly distributed over $[0, \pi]$, the number $n(\Delta \phi)$ of neurons in a population

$$
G_{k}=\left\{j \mid \phi_{i}+(k-0.5) \Delta \phi \leqslant \phi_{j}<\phi_{i}+(k+0.5) \Delta \phi\right\}
$$

increases linearly with $N$, i.e. $n(\Delta \phi)=N \Delta \phi / \pi$. Let us assume that $\Delta \phi$ is sufficiently small so that $J\left(\phi_{i}-\phi_{j}\right) \approx J\left(\phi_{i}-k \Delta \phi\right)$ for all neurons $j$ in population $G_{k}$. Since we are interested in the limit of a large number of neurons, we imagine now that we increase the number of neurons so that $n(\Delta \phi) \gg 1$ (i.e. $N \gg \pi / \Delta \phi)$. The number of spikes emitted by population $G_{k}$ in a short interval $\Delta t$ is

$$
\sum_{j \in G_{k}} \int_{t}^{t+\Delta t} \delta\left(t^{\prime}-t_{j}^{(f)}\right) \mathrm{d} t^{\prime} \approx n(\Delta \phi) A\left(\phi_{i}-k \Delta \phi, t\right) \Delta t
$$

where $A\left(\phi_{i}-k \Delta \phi, t\right)$ is the population activity of population $G_{k}$ (Gerstner 2000). We may therefore rewrite equation (21) in terms of the population activities

$$
h_{i}^{\mathrm{int}}(t)=\frac{1}{N} \sum_{k} J\left(\phi_{i}-k \Delta \phi\right) n(\Delta \phi) \int_{0}^{\infty} \epsilon(s) A\left(\phi_{i}-k \Delta \phi, t-s\right) \mathrm{d} s .
$$

As an aside we note that in the limit of $\Delta \phi \rightarrow 0$ equation (24) turns as expected into a continuum model

$$
h^{\mathrm{int}}(\phi, t)=\pi^{-1} \int_{0}^{\pi} J\left(\phi-\phi^{\prime}\right) \int_{0}^{\infty} \epsilon(s) A\left(\phi^{\prime}, t-s\right) \mathrm{d} s \mathrm{~d} \phi .
$$

In a stationary state, the average over the firing times of a large group of identical neurons (receiving the same constant stimulus) is equivalent to the temporal average of the spikes of a single neuron in that population (Gerstner 1995). In a stationary state, we may therefore identify the population activity with the neuronal firing rate

$$
A\left(\phi_{i}-k \Delta \phi\right) \equiv r\left(\phi_{i}-k \Delta \phi\right) .
$$

Hence, with $\int_{0}^{\infty} \epsilon(s) \mathrm{d} s=1$

$$
\begin{aligned}
h_{i}^{\mathrm{int}}(t) & =\frac{1}{N} \sum_{k} J\left(\phi_{i}-k \Delta \phi\right) n(\Delta \phi) r\left(\phi_{i}-k \Delta \phi\right) \\
& =\frac{1}{N} \sum_{j} J\left(\phi_{i}-\phi_{j}\right) r\left(\phi_{j}\right) .
\end{aligned}
$$

Note that the concept of discrete populations has only been used for the intermediate steps of the argument. In the transition from (27) to (28) we have replaced the sum over the populations by the original sum over all neurons. 


\section{References}

Abbott L F and Dayan P 1999 The effect of correlated variability on the accuracy of a population code Neural Comput. 11 91-101

Ben-Yishai R, Bar-Or R L and Sompolinsky H 1995 Theory of orientation tuning in visual cortex Proc. Natl Acad. Sci. USA $923844-8$

Blahut R E 1988 Principles and Practice of Information Theory (Cambridge, MA: Addison-Wesley)

Braitenberg V and Schuz A 1991 Anatomy of the Cortex: Statistics and Geometry (Berlin: Springer)

Brunel N and Nadal J P 1998 Mutual information, Fisher information and population coding Neural Comput. 10 1731-57

Camperi M and Wang X J 1998 A model of visuospatial working memory in prefrontal cortex: recurrent network and cellular bistability J. Comput. Neurosci. 5 383-405

Cox D R 1962 Renewal Theory (London: Meathuen)

Douglas R J, Koch C, Mahowald M, Martin K A C and Suarez H H 1995 Recurrent excitation in neocortical circuits Science 269 981-5

Ferster D 1986 Orientation selectivity of synaptic potentials in neurons of cat primary visual cortex J. Neurosci. 6 1284-301

Ferster D and Koch C 1987 Neuronal connections underlying orientation selectivity in cat visual cortex Trends Neurosci. 10 487-92

Fisher N 1993 Statistical Analysis of Circular Data (Cambridge: Cambridge University Press)

Georgopoulos A P, Caminit R, Kalaska J and Massey J 1983 Spatial coding of movements: a hypothesis concerning the coding movement direction by motor cortical populations Exp. Brain Res. 7 327-36

Gerstner W 1995 Time structure of the activity in neural network models Phys. Rev. E 51 738-58

2000 Population dynamics of spiking neurons: fast transients, asynchronous states, and locking Neural Comput. $1243-89$

Gerstner W and Van Hemmen J 1992 Associative memory in a network of spiking neurons Network 3 139-64

Hansel D and Sompolinsky H 1998 Modeling feature selectivity in local cortical circuits Methods in Neuronal Modeling ed C Koch and I Segev (Cambridge, MA: MIT Press) Chapter 13

Hubel D H and Wiesel T N 1968 Receptive fields and functional architecture of monkey striate cortex J. Physiol. (Lond.) 195 215-43

1974 Sequence regularity and geometry of orientation columns in the monkey striate cortex J. Comparative Neurol. 158 267-93

Laing C R and Chow C C 2001 Stationary bumps in networks of spiking neurons Neural Comput. 13 no 7

Mardia K 1972 Statistics of Directional Data (London: Academic)

Martin K 1988 From single cells to simple circuits in the cerebral cortex Q. J. Exp. Physiol. 73 637-702

O'Keefe L, Bair W and Movshon J A 1997 Response variability of MT neurons in macaque monkey Soc. Neurosci. Abstr. 231125

Plesser H E and Gerstner W 2000 Noise in integrate-and-fire neurons: from stochastic input to escape rates Neural Comput. 12 367-84

Pouget A, Deneve S, Ducom J C and Latham P 1999 Narrow vs wide tuning curves: what's best for a population code Neural Comput. 11 85-90

Redish A, Elga A and Touretzky D 1996 A coupled attractor model of the rodent head direction system Network 7 671-85

Salinas E and Abbott L 1994 Vector reconstruction from firing rates J. Comput. Neurosci. 1 89-107

Schiller P H, Finlay B L and Volman S F 1976 Short-term response variability of monkey striate neurons Brain Res. 105 347-9

Shadlen M and Newsome W T 1998 The variable discharge of cortical neurons: implications for connectivity, computation, and information coding J. Neurosci. 18 3870-96

Snippe H P and Koenderink J J 1992 Information in channel-coded systems: correlated receivers Biol. Cybern. 67 183-90

Somers D C, Nelson S B and Sur M 1995 An emergent model of orientation selectivity in cat visual cortical simple cells J. Neurosci. 15 5448-65

Spiridon M 2000 Signal transmission reliability in densely connected networks of spiking neurons PhD Thesis Swiss Federal Institute of Technology, Lausanne

Vogels R, Spileers W and Orban G A 1989, The response variability of striate cortical neurons in the behaving monkey Exp. Brain Res. 77 432-6

Wehmeier U, Dong D, Koch C and Essen D V 1998 Modeling the mammalian visual system Methods in Neuronal Modeling ed C Koch and I Segev (Cambridge, MA: MIT Press) pp 335-59 
Werner G and Mountcastle V B 1965 The variability of central neural activity in a sensory system and its implications for the central reflection of sensory events J. Neurophysiol. 26 958-77

Wörgötter F and Koch C 1991 A detailed model of the primary visual pathway in the cat: comparison of afferent excitatory and intracortical inhibitory connection schemes for orientation selectivity J. Neurosci. 11 1959-79

Yoon H and Sompolinsky H 1998 The effect of correlations on the Fisher information of population code Advances in Neural Information Processing Systems vol 11 ed M I Jordan, M J Kearns and S A Solla (Cambridge, MA: MIT Press) pp 167-73

Zohary E, Shadlen M and Newsome W 1994 Correlated neuronal discharge rate and its implications for psychophysical performance Nature 370 140-3 